\title{
A STUDY ON DYSLIPIDAEMIA IN CHRONIC KIDNEY DISEASE (CKD) WITH SPECIAL REFERENCE TO HAEMODIALYSIS
}

\author{
Shweta Sharma1 ${ }^{1}$ Sandhya Gautam², Dhanveer Singh ${ }^{3}$, Prachi Sharma 4 , Avriti Baweja ${ }^{5}$ \\ ${ }_{1}^{1}$ Assistant Professor, Department of Medicine, LLRMMC. \\ ${ }^{2}$ Assistant Professor, Department of Medicine, LLRMMC. \\ ${ }^{3}$ Assistant Professor, Department of Medicine, Subharti Medical College. \\ 4Junior Resident, Department of Medicine, LLRMMC. \\ 5Junior Resident, Department of Medicine, LLRMMC.
}

\section{ABSTRACT}

Dyslipidaemia is a common complication of CKD. It is also an independent predictor of cardiovascular disease, which is a major cause of mortality in these patients.

\section{AIMS AND OBJECTIVES \\ - To study the levels of lipids in CKD. \\ - $\quad$ Effect of haemodialysis on lipids.}

\section{MATERIALS AND METHODS}

250 patients of CKD were screened and out of them 118 patients with chronic renal failure associated with dyslipidaemia completed the study. Lipid variables were measured before and after 6 months of regular biweekly bicarbonate-based haemodialysis. Lipid variables were compared with 50 age and sex matched healthy controls. All the values were expressed in terms of mean and standard deviation and statistical analysis was carried out by using student ' $\mathrm{T}$ ' test.

\section{RESULTS}

We found that the cholesterol level was maximally deranged followed by LDL and followed by triglycerides in the study group as compared to controls. There was reduction in total cholesterol, triglycerides, LDL, VLDL, while increment in mean HDL after dialysis. But the changes were statistically insignificant.

\section{CONCLUSION}

CKD is associated with dyslipidaemia even in younger age groups. The reduction in lipid profile was insignificant even after 6 months of haemodialysis and $\mathrm{p}$ value remained $>0.05$.

\section{KEYWORDS}

Kidney, Lipid, Cholesterol, Triglycerides, LDL, HDL, VLDL, Cardiovascular Risk, Haemodialysis, Diabetes, Hypertension.

HOW TO CITE THIS ARTICLE: Sharma S, Gautam S, Singh D, et al. A study on dyslipidaemia in chronic kidney disease (CKD) with special reference to haemodialysis. J. Evolution Med. Dent. Sci. 2016;5(28):1433-1437, DOI: 10.14260/jemds/2016/337

\section{INTRODUCTION}

CKD refers to a spectrum of the varied pathophysiologic processes associated with abnormal kidney function and a progressive decline in Glomerular Filtration Rate (GFR) for 3 months or more.(1) 3-5.(1)

The term chronic renal failure corresponds to CKD stages

It is known that patients with impaired renal function exhibit significant alterations in lipoprotein metabolism.(2) which in their advanced form may result in the development of severe dyslipidaemia.

Dyslipidaemia, in turn, along with other co-existing risk factors adds to the risk of cardiovascular diseases in CKD.(3) which is a major cause of mortality in these patients. ${ }^{(4)}$

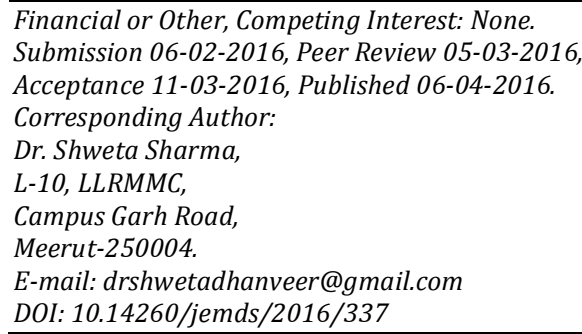

Hypertriglyceridemia and increased LDL in CKD are consequences of increased production as well as decreased catabolism of the same.(5)

The increased production is due to impaired carbohydrate tolerance and increased hepatic VLDL synthesis.(2) The reduced catabolism is due to decreased activity of hepatic lipase and endothelial lipoprotein lipase.(6)

There is reduced LCAT activity in CKD.(6) resulting in reduced reverse cholesterol transport and hence increased atherogenesis.

\section{AIMS AND OBJECTIVES}

1. To study the levels of lipids in CKD.

2. To study the effect of haemodialysis on lipids.

\section{MATERIALS AND METHODS}

This prospective study was done on 118 patients suffering from CKD with CRF associated with dyslipidaemia attending medicine OPD or admitted indoor at LLRM Medical College and Subharti Medical College and their associated hospitals between 2013 and 2015. The study protocol was approved by the Ethical Board of each participating site. All patients gave their written informed consent before entering the study. 


\section{Inclusion Criteria}

1. Age $>15$ years.

2. CKD (According to KDIGO guidelines 2012) with CRF

3. Dyslipidaemia.

\section{Exclusion Criteria}

1. Genetic dyslipidaemia.

2. Obesity with hyperlipidaemia.

3. Cholestatic liver disease.

4. Hypothyroidism.

5. Patients with history of smoking or alcohol intake.

6. Patients receiving anabolic steroids or oestrogen before the study.

250 patients of CKD were screened, out of which 120 were found to have CRF with dyslipidaemia, considering the inclusion and exclusion criteria.

12 patients left the study in between due to change of residence, death or other personal reasons; 118 patients completed the study. GFR was estimated by using MDRD formula. Their total cholesterol, TG, HDL, LDL, VLDL were measured.

Lipid variable were compared with 50 age and sex matched controls.

Controls were healthy people who were asymptomatic with normal lipid profile, kidney profile, CVS profile, no history of alcohol intake and having normal BMI. Patients were subjected to twice weekly haemodialysis using bicarbonate based fluid. Lipid profile was compared before and after 6 months of regular biweekly haemodialysis. Statistical analysis was carried out using paired student's ' $\mathrm{T}$ ' test.

\section{OBSERVATIONS AND RESULTS}

Among the 118 patients 70 (59\%) were males, while 48 (41\%) were females.

\begin{tabular}{|c|c|}
\hline Sex & Number \\
\hline Males & 70 \\
\hline Females & 48 \\
\hline \multicolumn{2}{|c|}{ Table 1: Sex distribution of cases } \\
\hline
\end{tabular}

Majority of them, 68 (57.6\%) were in the age group of 3160 years, while $15(12.7 \%)$ were below 30 years of age and 35 $(29.7 \%)$ were above 60 years of age.

\begin{tabular}{|c|c|}
\hline Age & Number \\
\hline$<30$ & 15 \\
\hline $31-60$ & 68 \\
\hline$>60$ & 35 \\
\hline \multicolumn{2}{|c|}{ Table 2: Age distribution of cases } \\
\hline
\end{tabular}

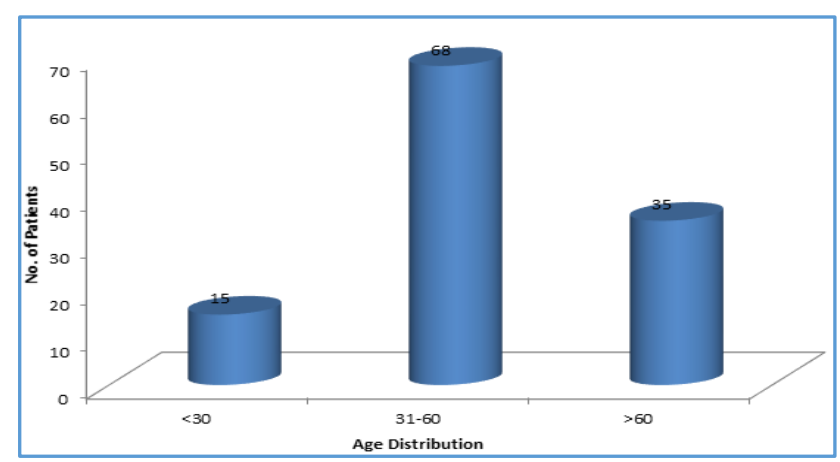

74 (63\%) patients were in CKD stage 5 and 44 (37\%) were in stage 4.

\begin{tabular}{|c|c|}
\hline Stage & Number \\
\hline 4 & 44 \\
\hline 5 & 74 \\
\hline \multicolumn{2}{|c|}{ Table 3: Stages of CKD } \\
\hline
\end{tabular}

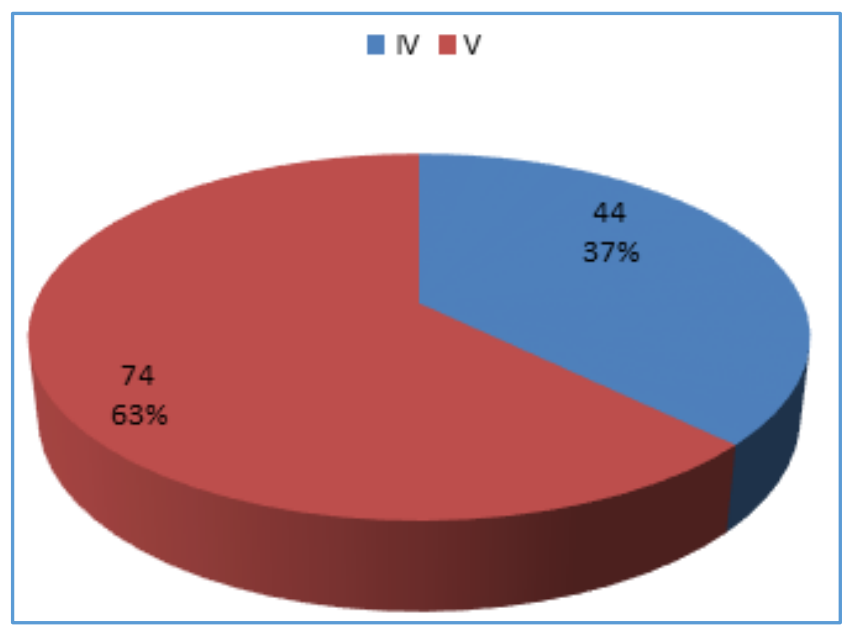

$38(32.2 \%)$ patients were diabetic and $76(64.4 \%)$ were hypertensive.

- Among the diabetics $(\mathrm{n}=38) 13(34.2 \%)$ were in stage 4 , while $25(65.8 \%)$ were in stage 5 CKD.

- Among the non-diabetics $(n=80) 31(38.8 \%)$ were in stage 4 , while 49 (61.2\%) were in stage 5 CKD.

- Among the hypertensives ( $n=76) 22$ (29\%) were in stage 4 , while $54(71 \%)$ were in stage 5 CKD.

- Among the non-hypertensives $(n=42) 22$ (52.3\%) were in stage 4 , while $20(47.7 \%)$ were in stage 5 CKD.

The values of lipid profile in controls $(n=25)$ were as follows

\begin{tabular}{|c|c|c|c|}
\hline Lipids & Range & Mean & SD \\
\hline Cholesterol & $88(102-190)$ & 162.3 & 27.16 \\
\hline Triglyceride & $48(98-146)$ & 128.3 & 15.65 \\
\hline HDL & $44(42-86)$ & 56.13 & 11.33 \\
\hline LDL & $46(46-92)$ & 73.73 & 16.59 \\
\hline VLDL & $12(16-28)$ & 23.21 & 3.92 \\
\hline \multicolumn{4}{|c|}{ Table 4: Lipid Profile in Controls } \\
\hline
\end{tabular}

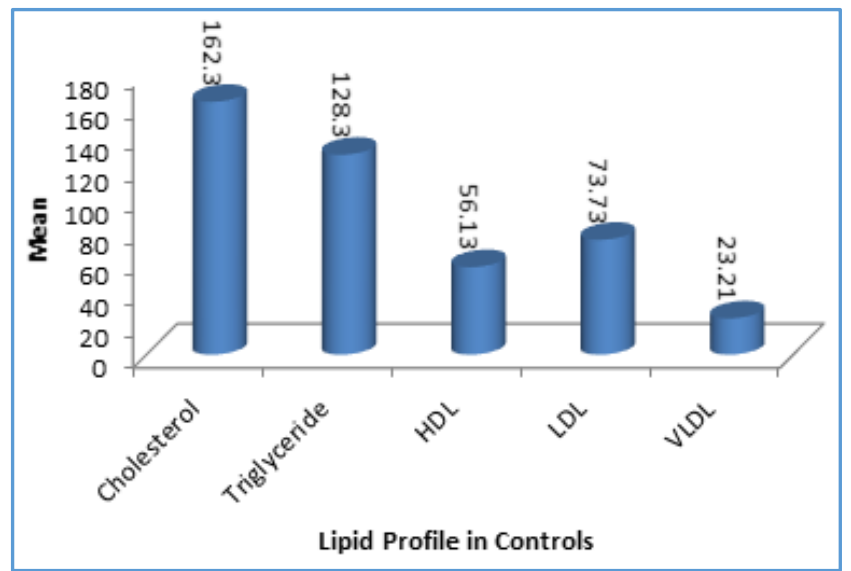

Mean blood urea and serum creatinine in the control group were $28.73 \mathrm{mg} \%$ and $0.68 \mathrm{mg} \%$ respectively.

In the study group, mean value of blood urea and serum creatinine at the time of presentation were $200.16 \mathrm{mg} \%$ [range (76-327)] and $9.05 \mathrm{mg} \%$ [Range (2.7-24)] respectively. 


\begin{tabular}{|c|c|c|c|c|}
\hline Stage & Lipid Profile & Range & Mean & SD \\
\hline \multirow{4}{*}{ IV (n=13) } & Cholesterol & $40[198-238]$ & 211.46 & 10.88 \\
\cline { 2 - 5 } & Triglyceride & $50[132-182]$ & 155.00 & 12.50 \\
\cline { 2 - 5 } & HDL & $10[28-38]$ & 34.0 & 2.70 \\
\cline { 2 - 5 } & LDL & $66[102-168]$ & 142.69 & 19.60 \\
\cline { 2 - 5 } & VLDL & $16[30-46]$ & 34.46 & 4.01 \\
\hline \multirow{4}{*}{ V (n=25) } & Cholesterol & $60[196-256]$ & 212.57 & 13.95 \\
\cline { 2 - 5 } & Triglyceride & $48[134-182]$ & 157.82 & 11.46 \\
\cline { 2 - 5 } & HDL & $22[26-48]$ & 37.14 & 6.34 \\
\cline { 2 - 5 } & LDL & $86[100-186]$ & 142.6 & 19.18 \\
\cline { 2 - 5 } & VLDL & $19[29-48]$ & 35.08 & 4.48 \\
\hline \multicolumn{5}{|c|}{ Table 5: Lipid Profile in Diabetics in } \\
\multicolumn{5}{|c|}{ Relation to Stage (n=38) } \\
\hline
\end{tabular}

\begin{tabular}{|c|c|c|c|c|}
\hline Stage & Lipid Profile & Range & Mean & SD \\
\hline \multirow{4}{*}{ IV (n=22) } & Cholesterol & $38(198-236)$ & 210 & 11.34 \\
\cline { 2 - 5 } & Triglyceride & $54(128-182)$ & 152.08 & 14.02 \\
\cline { 2 - 5 } & HDL & $10(28-38)$ & 35.75 & 2.8 \\
\cline { 2 - 5 } & LDL & $67(101-168)$ & 140.42 & 20.12 \\
\cline { 2 - 5 } & VLDL & $18(28-46)$ & 33.16 & 4.78 \\
\hline \multirow{4}{*}{ V (n=54) } & Cholesterol & $56(196-252)$ & 211.54 & 13.26 \\
\cline { 2 - 5 } & Triglyceride & $43(133-176)$ & 153.09 & 10.81 \\
\cline { 2 - 5 } & HDL & $24(24-48)$ & 38.72 & 6.38 \\
\cline { 2 - 5 } & LDL & $86(100-186)$ & 141.83 & 19.08 \\
\cline { 2 - 5 } & VLDL & $20(28-48)$ & 36.37 & 16.72 \\
\hline Table 7: Lipid Profile in Hypertensives in Relation to Stage \\
\hline \multicolumn{4}{|c|}{}
\end{tabular}
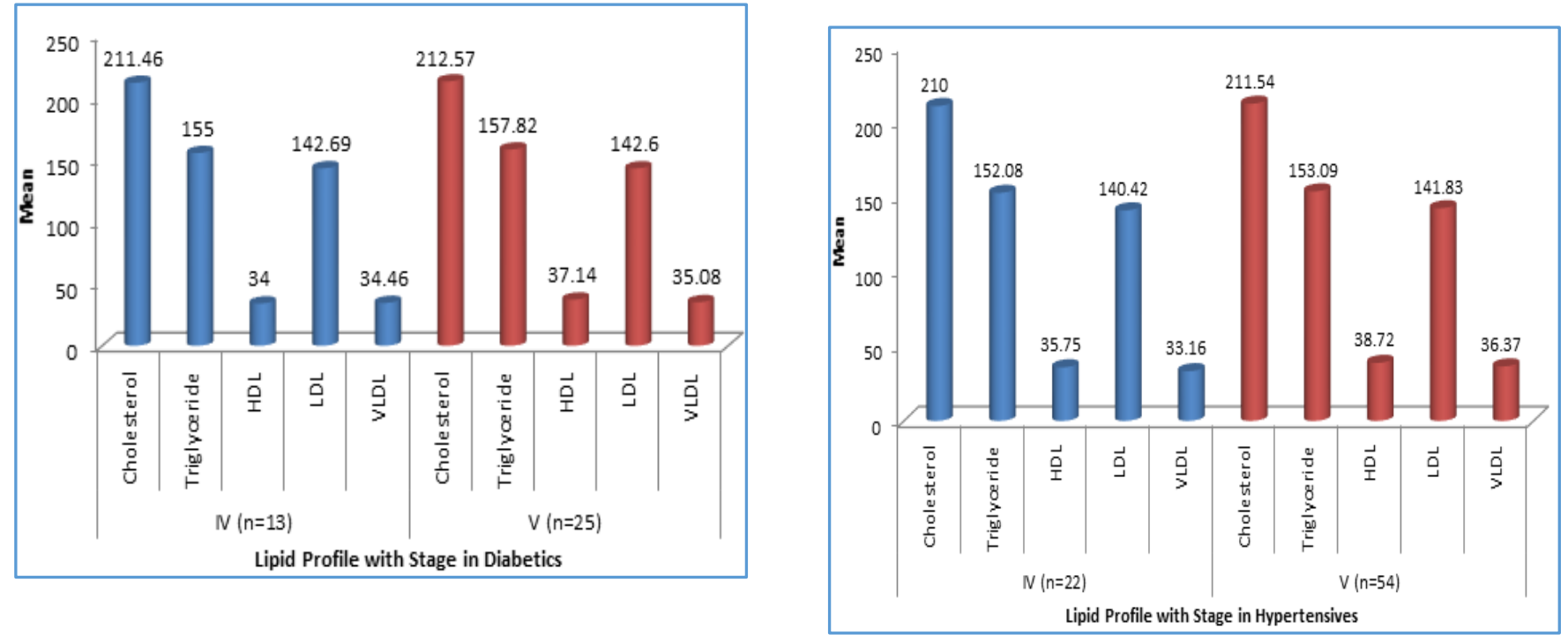

\begin{tabular}{|c|c|c|c|c|}
\hline Stage & Lipid Profile & Range & Mean & SD \\
\hline \multirow{4}{*}{ IV (n=31) } & Cholesterol & $45(189-234)$ & 209.46 & 15.94 \\
\cline { 2 - 5 } & Triglyceride & $48(124-172)$ & 153.30 & 13.0 \\
\cline { 2 - 5 } & HDL & $20(20-48)$ & 35.15 & 6.21 \\
\cline { 2 - 5 } & LDL & $50(118-168)$ & 150.23 & 15.44 \\
\cline { 2 - 5 } & VLDL & $13(29-42)$ & 35.30 & 3.35 \\
\hline \multirow{4}{*}{ V (n=49) } & Cholesterol & $48(198-246)$ & 210.97 & 11.66 \\
\cline { 2 - 5 } & Triglyceride & $44(132-176)$ & 157.66 & 12.20 \\
\cline { 2 - 5 } & HDL & $26(22-48)$ & 37.05 & 6.95 \\
\cline { 2 - 5 } & LDL & $64(104-168)$ & 144.28 & 17.63 \\
\cline { 2 - 5 } & VLDL & $13(29-42)$ & 35.28 & 2.98 \\
\hline Table 6: Lipid Profile in Non-Diabetics in Relation to Stage \\
\hline \multicolumn{4}{|c|}{} \\
\cline { 2 - 5 }
\end{tabular}

\begin{tabular}{|c|c|c|c|c|}
\hline Stage & Lipid Profile & Range & Mean & SD \\
\hline \multirow{5}{*}{ IV $(n=22)$} & Cholesterol & $40(198-238)$ & 208.23 & 15.8 \\
\hline & Triglyceride & $42(128-170)$ & 149.62 & 12.3 \\
\hline & HDL & $25(23-48)$ & 36.69 & 6.1 \\
\hline & LDL & $59(103-162)$ & 147.3 & 13.9 \\
\hline & VLDL & $11(27-38)$ & 33.23 & 3.77 \\
\hline \multirow{5}{*}{$V(n=20)$} & Cholesterol & $36(198-234)$ & 208.95 & 10.67 \\
\hline & Triglyceride & $44(130-174)$ & 153.46 & 11.38 \\
\hline & HDL & $25(23-48)$ & 37.18 & 6.5 \\
\hline & LDL & $60(102-162)$ & 141.26 & 16.9 \\
\hline & VLDL & $12(27-39)$ & 33.35 & 2.61 \\
\hline
\end{tabular}
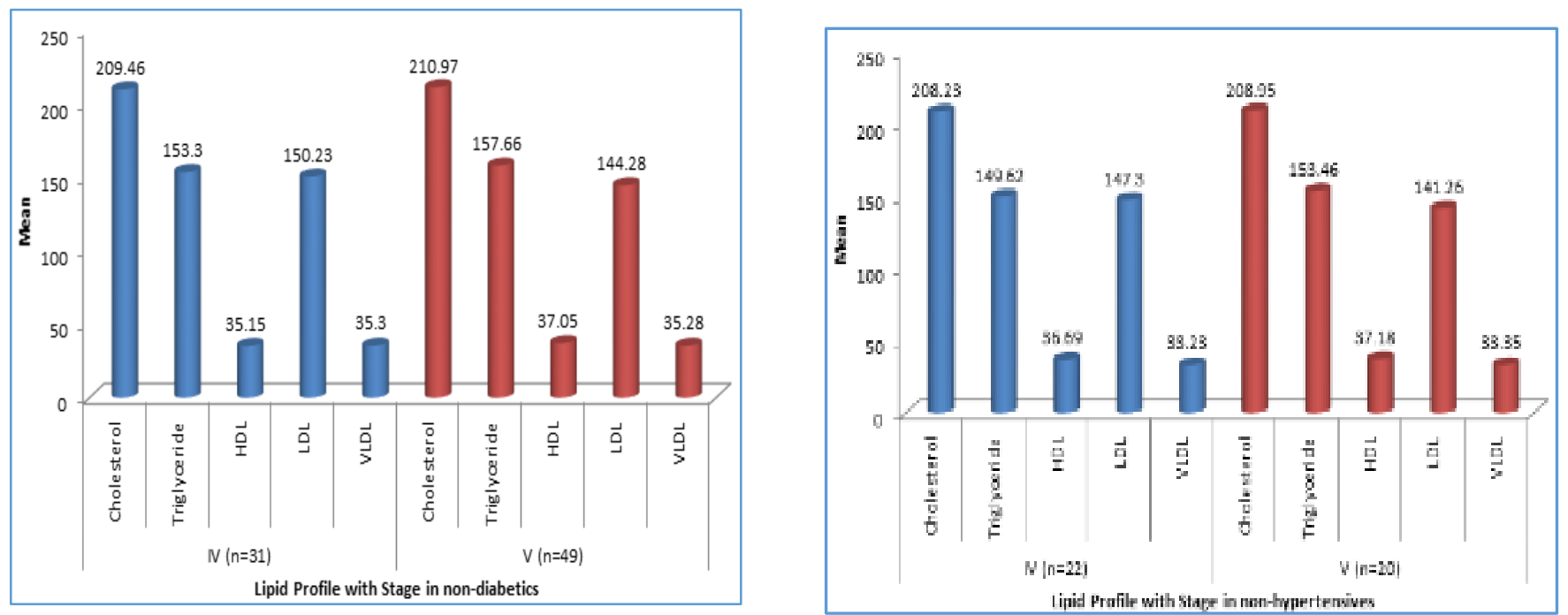
These values were compared with the values after 6 months of twice weekly bicarbonate-based haemodialysis.

\begin{tabular}{|c|c|c|c|}
\hline Lipid Profile & Range & Mean & SD \\
\hline Cholesterol & $48(188-236)$ & 208.73 & 0.7 \\
\hline Triglyceride & $52(124-176)$ & 149.46 & 0.54 \\
\hline HDL & $20(28-48)$ & 35.15 & 0.23 \\
\hline LDL & $67(101-168)$ & 144.42 & 16.97 \\
\hline VLDL & $8(28-36)$ & 32.07 & 2.36 \\
\hline \multicolumn{4}{|c|}{ Table 9: The Values of Lipids in Stage 4 } \\
Patients Post Dialysis (n=44) \\
\hline
\end{tabular}

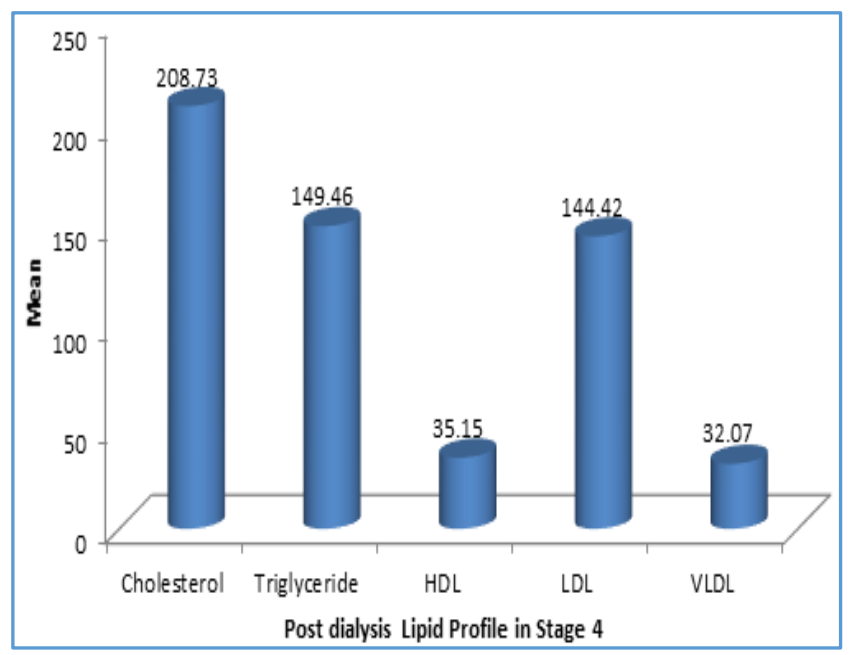

\begin{tabular}{|c|c|c|c|}
\hline Lipid Profile & Range & Mean & SD \\
\hline Cholesterol & $56(196-252)$ & 210.25 & 12.64 \\
\hline Triglyceride & $44(128-172)$ & 151.3 & 10.20 \\
\hline HDL & $25(23-48)$ & 37.12 & 6.35 \\
\hline LDL & $88(98-186)$ & 141.4 & 17.94 \\
\hline VLDL & $14(26-40)$ & 33.74 & 2.35 \\
\hline \multicolumn{3}{|c|}{ Table 10: The Values of Lipids in Stage } \\
5 Patients post dialysis (n=74) \\
\hline
\end{tabular}

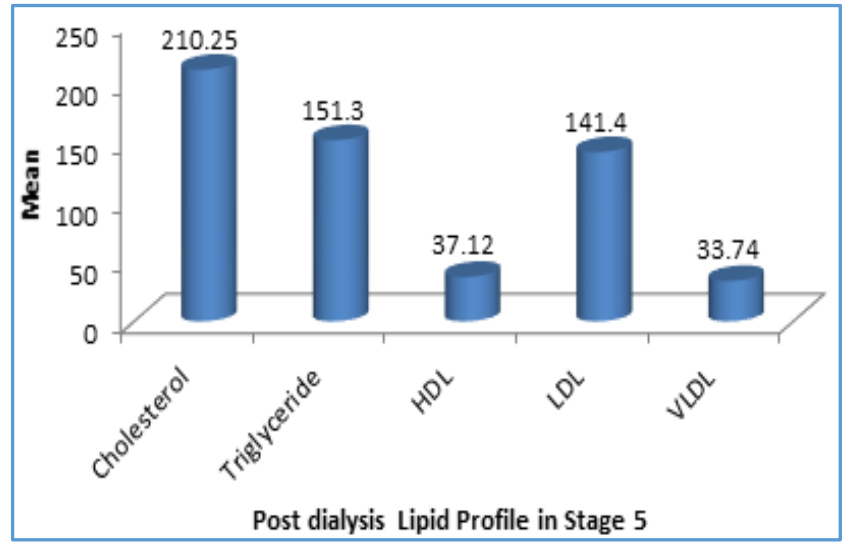

It was found that the lipid profile was deranged significantly in patients of CKD as compared to controls $(\mathrm{p}<0.05)$.

It was seen that TC levels were maximally deranged, followed by LDL and followed by TG levels when compared to the control group.

Also the derangement was more in stage 5 CKD in all the 4 groups (DM, non-DM, HTN, non-HTN) as compared to stage 4 but the difference was not statistically significant ( $p>0.05$ ). Also when diabetics were compared with non-diabetics, derangement was more in DM but with no statistically significant difference $(\mathrm{p}>0.05)$.

The values were comparable in both hypertensive and non-hypertensive groups.

But after 6 months of regular twice weekly haemodialysis, it was found that in all the subgroups, some decrement occurred in the values of total cholesterol, TG, LDL, VLDL and a pattern of increment was present in HDL values but these values when statistically compared, were found to be insignificant ( $\mathrm{p}>0.05$ ).

Post dialysis mean values of blood urea and serum creatinine were $90.64 \mathrm{mg} \%$ and $5.64 \mathrm{mg} \%$ respectively.

\begin{tabular}{|c|c|c|c|c|c|c|c|}
\hline \multirow[t]{2}{*}{ Lipid Profile } & \multicolumn{3}{|c|}{ Pre-Dialysis } & \multicolumn{3}{|c|}{ Post Dialysis } & \multirow{2}{*}{ P-value } \\
\hline & Range & Mean & SD & Range & Mean & SD & \\
\hline Cholesterol & 49 & 210 & 13.41 & 48 & 208.73 & 13.11 & 0.73 \\
\hline Triglyceride & 58 & 154.15 & 12.53 & 52 & 149.46 & 12.1 & 0.17 \\
\hline HDL & 20 & 34.57 & 4.73 & 20 & 35.15 & 4.67 & 0.74 \\
\hline LDL & 66 & 146.46 & 17.71 & 67 & 144.42 & 16.97 & 0.67 \\
\hline VLDL & 17 & 34.46 & 3.64 & 8 & 33.07 & 2.96 & 0.07 \\
\hline
\end{tabular}

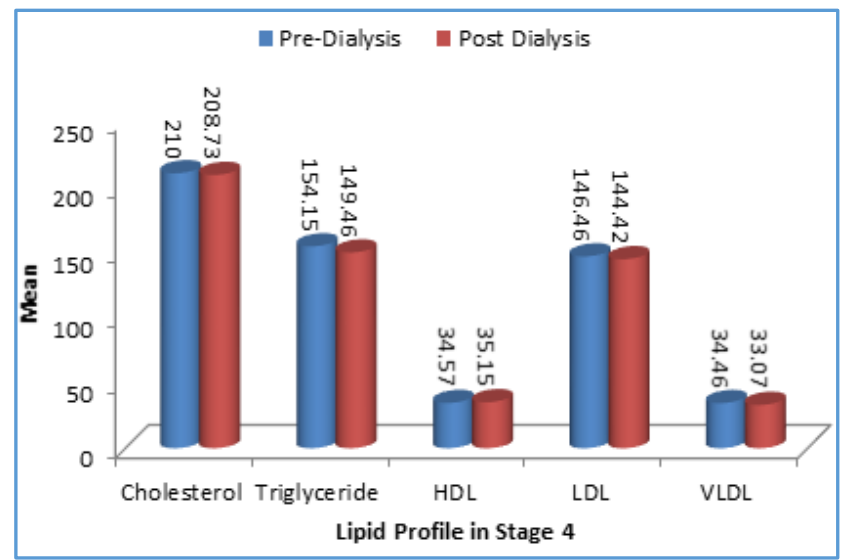

\begin{tabular}{|c|c|c|c|c|c|c|c|}
\hline $\begin{array}{c}\text { Lipid } \\
\text { Profile }\end{array}$ & \multicolumn{3}{|c|}{ Pre-Dialysis } & \multicolumn{3}{c|}{ Post Dialysis } & \multirow{2}{*}{$\begin{array}{c}\text { P- } \\
\text { value }\end{array}$} \\
\hline & Range & Mean & SD & Range & Mean & SD & \\
\hline Cholesterol & 60 & 211.72 & 12.73 & 56 & 210.25 & 12.64 & 0.48 \\
\hline Triglyceride & 50 & 157.74 & 11.78 & 44 & 151.3 & 10.20 & 0.06 \\
\hline HDL & 26 & 37.09 & 6.62 & 25 & 37.12 & 6.35 & 0.97 \\
\hline LDL & 86 & 143.5 & 18.27 & 88 & 141.4 & 17.94 & 0.48 \\
\hline VLDL & 19 & 35.18 & 3.74 & 14 & 33.74 & 3.35 & 0.07 \\
\hline Table 12: Net Variation in Lipid before and after Dialysis in Stage 5 (n=74) \\
\hline
\end{tabular}




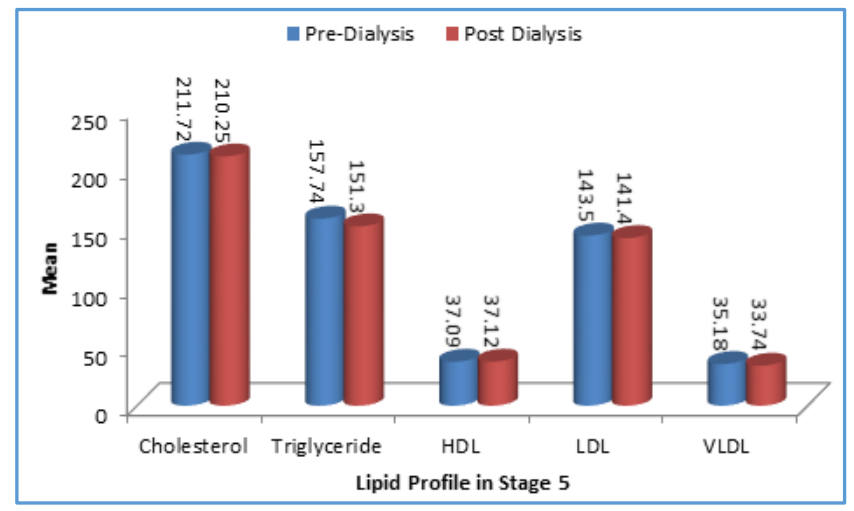

\section{SUMMARY}

CKD is a significant health problem. Dyslipidaemia adds to the already high cardiovascular risk in these patients. Several studies have found a positive correlation between CRF and dyslipidaemia.(7)

So it is important to know whether haemodialysis, the main modality of management in patients with CRF also takes care of this culprit or not.

In view of this, lipid profile was compared in CRF patients before and after 6 months of regular biweekly bicarbonate-based haemodialysis.

The lipid profile was found to be significantly deranged in the cases as compared to the controls. It was also seen that although some changes towards improvement occurred after haemodialysis, i.e. decrease in TC, TG, LDL, VLDL and an increase in $\mathrm{HDL}$, but these changes were statistically insignificant.

Along with there was significant reduction in the mean value of blood urea and serum creatinine post dialysis.

Our results are similar to the study done by Tsimihodimos et al.(8) and Babazano et al.(9) where they found that there was no significant change in lipid levels post dialysis. But this was in contrast to a study by Mitwalli et al.(10) in 2011, where they found that despite regular haemodialysis, dyslipidaemia progressed.

\section{CONCLUSION}

Dyslipidaemia is another black hole in the dark world of CKD. Moreover, only haemodialysis which is a part and parcel of ESRD management does not take full care of this issue.
So, additional measures are required in this direction and should be a matter of concern.

\section{REFERENCES}

1. Joanne M Bargman, Karl S Korecki. Chronic kidney disease. Harrison's principles of internal medicine. Mc Graw Hill, 18th ed;Vol 2:2308-2321.

2. Bonnie $\mathrm{CH}$ Kwan, Florian Kronenberg, Srinivasan Beddhu, et al. Lipoprotein metabolism and lipid management in chronic kidney disease. Journal of the american society of nephrology 2007;18:1246-1261.

3. Variri ND. Dyslipidemia of chronic renal failure: the nature, mechanisms and potential consequences. Am J Physiology Renal Physiology 2006;290:262-272.

4. Adrian D Slee. Exploring metabolic dysfunction in chronic kidney disease. Nutrition and metabolism 2012;9(1):36.

5. Omran J. Dyslipidemia in patients with chronic and endstage kidney disease. Cardiorenal Med 2013;3:165-177.

6. Sarnak MJ, Levey AS, Schoolwerth AC, et al. Kidney disease as a risk factor for development of cardiovascular disease: a statement from the American heart association councils on kidney in cardiovascular disease, high blood pressure research, clinical cardiology and epidemiology and prevention. Circulation 2003;108(17):2154-69.

7. Ponnudhali D, Nagarajan P. Lipoprotein (a) and dyslipidemia in predialysis chronic kidney disease patients and in patients of maintainance hemodialysis. International Journal of Basic Medical Science 2011;2(5):131-137.

8. Vasilis Tsimihodimos, Zoi Mitrogianni, Moses Elisaf. Dyslipidemia associated with chronic kidney disease. The Open Cardiovascular Medicine Journal 2011;5:4148.

9. Babazono $T$, Nakamoto $H$, Kasai $K$, et al. Effects of icodextrin on glycemic and lipid profiles in diabetic patients undergoing peritoneal dialysis. Am J Nephrol 2007;27:401-15.

10. Mitwalli AH, Alam AA, Al Wakeel JS, et al. Dyslipidemia in dialysis patients. Saudi J Kidney Dis Transpl 2011;22(4):689-94. 